\title{
Molecular dynamics of polarizable point dipole models for molten Nal. Comparison with first principles simulations.
}

\author{
V. Bitrián, O. Alcaraz, and J. Trullàs \\ Departament de Física i Enginyeria Nuclear, Universitat Politècnica de Catalunya, Campus Nord 08034 Barcelona, Spain
}

\begin{abstract}
Molecular dynamics simulations of molten NaI at $995 \mathrm{~K}$ have been carried out using polarizable ion models based on rigid ion pair potentials to which the anion induced dipole polarization is added. The polarization is added in such a way that point dipoles are induced on the anions by both local electric field and deformation short-range damping interactions that oppose the electrically induced dipole moments. The structure and self-diffusion results are compared with those obtained by Galamba and Costa Cabral using first principles Hellmann-Feynman molecular dynamics simulations and using classical molecular dynamics of a shell model which allows only the iodide polarization.
\end{abstract}

\section{Introduction}

Polarization effects are of great interest in understanding the structure and dynamics of ionic systems. Since NaI is an alkali halide with a high anionic polarizability, it has been widely studied by classical molecular dynamics simulations of polarizable ion models. The earliest molecular dynamics (MD) simulations of polarizable ions were carried out using shell models (SM) [1,2]. The dipoles of SM are represented by a pair of point charges, namely, a positive core and a negative shell joined by a harmonic spring. Recently, molten $\mathrm{NaI}$ [3], as well as molten silver halides ( $\mathrm{AgCl}, \mathrm{AgBr}$ and $\mathrm{AgI}$ ) [4-6], have also been simulated using polarizable point dipole models (PPDM), in which a point inducible dipole is added on each ionic site. Both SM and PPDM are based on rigidion models (RIM) where the ions interact solely through effective pair potentials. Moreover, Galamba and Costa Cabral [7] have carried out first principle HellmanFeynman molecular dynamics (HFMD) of molten $\mathrm{NaI}$ at temperature close to the melting point, and compared the structure and self diffusion results with those obtained using classical molecular dynamics of the SM proposed by Dixon and Sangster [1]. This SM allows only the iodide polarization, and the parameter values of the rigid ion pair potential are those given in Table 2-I of reference [1]. Their work prompted us to carry out MD simulations of molten NaI by using a PPDM based on the same rigid ion pair potential. In this paper we compare the structure and self-diffusion results for the PPDM, as well as for the RIM on which the former is constructed, with those for HFMD and SM obtained by Galamba and Costa Cabral.

\section{Simulated models}

We have carried out MD simulations of molten NaI using a rigid ion model (RIM) and a polarizable point dipole model (PPDM).

The ions of the RIM interact solely through the effective pair potential of the functional form of BornMayer-Huggins [2]

$\phi_{a b}(r)=\frac{e^{2} z_{a} z_{b}}{r}+B_{a b} \exp \left[\gamma\left(\sigma_{a b}-r\right)\right]-\frac{C_{a b}}{r^{6}}-\frac{D_{a b}}{r^{8}}$

with the values of the parameters proposed by Dixon and Sangster in Table 2-I of reference [1], which are those used by Galamba and Costa Cabral [7] to simulate the SM.

The anions of the PPDM have a polarizable point dipole whose moment is

$\mathbf{p}_{i}=\alpha \mathbf{E}_{i}+\mathbf{p}_{i}^{s}=\alpha \mathbf{E}_{i}-\alpha \sum_{j \neq i}^{N} f\left(r_{i j}\right) \frac{z_{j} e}{r_{i j}^{3}} \mathbf{r}_{i j}$

where $\alpha=6.24 \AA^{3}$ is the iodide polarizability [8] (the same value as in [7]), $\mathbf{E}_{i}$ is the local electric field at position $\mathbf{r}_{i}$, and $\mathbf{p}_{i}^{s}$ is the short-range damping contribution due to the neighboring ions. If this damping term is omitted the iodides polarize catastrophically. Following Wilson and Madden [9], the damping function in (2) is the Tang and Toennies dispersion function [10]

$f(r)=\exp (-r / \rho) \sum_{k=0}^{4} \frac{(r / \rho)^{k}}{k !}$ 
where we assume $\rho=0.36 \AA$. The many-body induced polarization interactions are added to the rigid ion contributions of $\phi_{a b}(r)[3]$.

\section{Results}

\subsection{Structure}

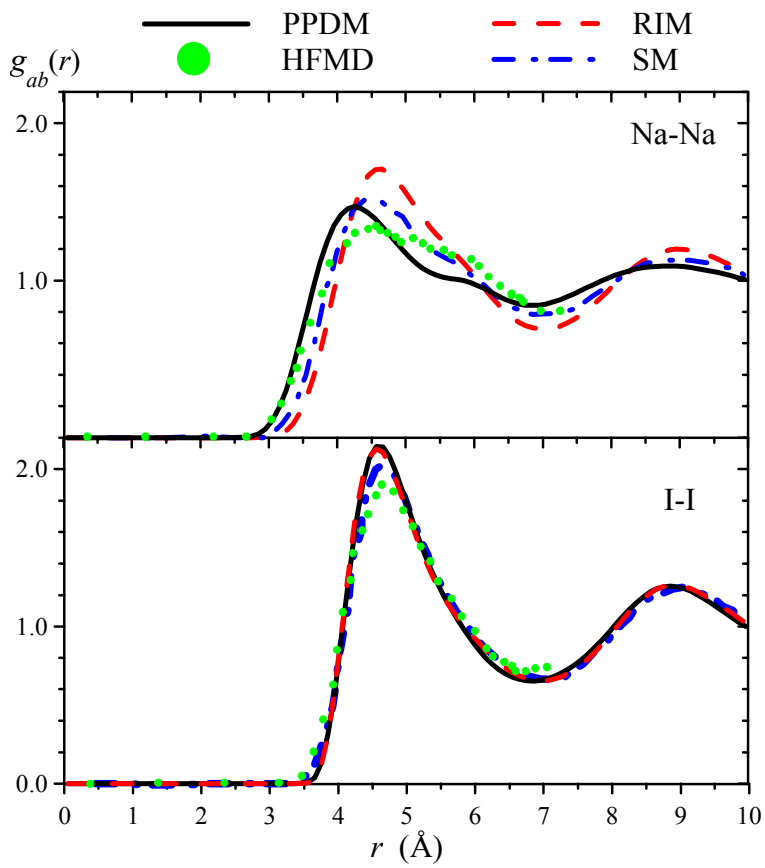

Fig. 1. Radial distribution functions between like ions $g_{\mathrm{NaNa}}(r)$ (top) and $g_{\text {III }}(r)$ (bottom), for RIM (red dashed line), PPDM (black solid line), SM (blue dash and dot line) and HFMD (green circles)

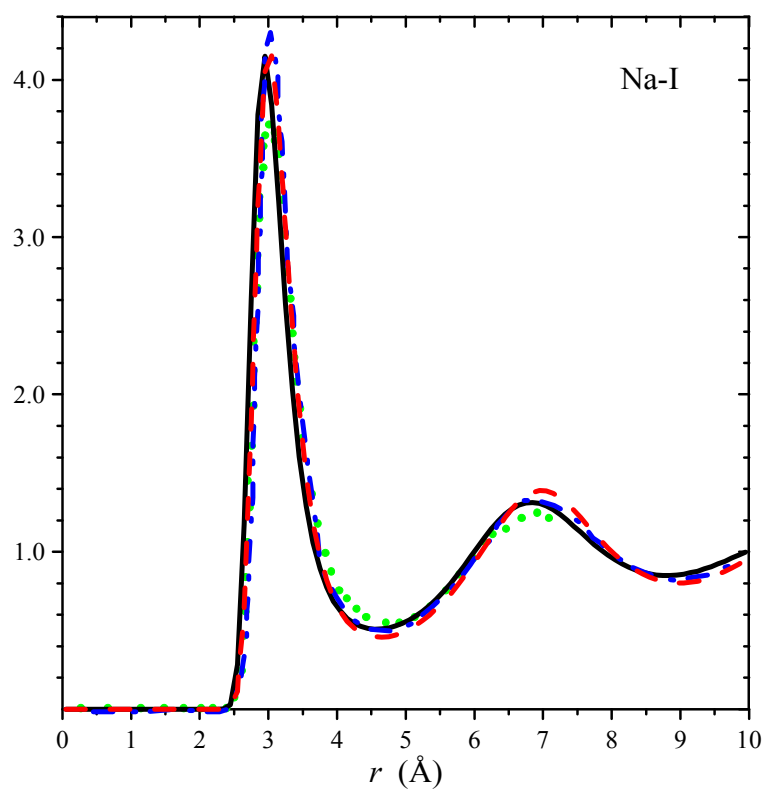

Fig. 2. Radial distribution functions between unlike ions $g_{\mathrm{NaI}}(r)$, for RIM (red dashed line), PPDM (black solid line), SM (blue dash and dot line) and HFMD (green circles).
The main differences between the structures predicted by the RIM, PPDM, SM and HFMD for molten NaI concern the cation-cation correlations. The peaks and valleys of $g_{\mathrm{II}}(r)$ and $g_{\mathrm{NaI}}(r)$ are almost at the same position for the four sets of results, with the first peak of $g_{\mathrm{NaI}}(r)$ at about $3.0 \AA$ and that of $g_{\text {II }}(r)$ at $4.6 \AA$. However, while the $g_{\mathrm{NaNa}}(r)$ for RIM is in phase with $g_{\mathrm{II}}(r)$, those for PPDM, SM and HFMD are not in phase. The first peak of the $g_{\mathrm{NaNa}}(r)$ for PPDM is at about $4.2 \AA$, whereas that for $\mathrm{SM}$ and HFMD is at about $4.4 \AA$. The $g_{\mathrm{NaNa}}(r)$ for PPDM, SM and HFMD present a shoulder at about $5.8 \AA$, between the first and second peaks of $g_{\mathrm{NaI}}(r)$, i.e., around each cation there is a double shell of cations between the first and second shells of anions. The induced polarization in the anions screens the repulsion between cations in such a way that the separation between two cations can be smaller than it would be if the anions were not polarized, and the first shell of cations around a cation in RIM splits into a double shell of cations in polarizable ion models.

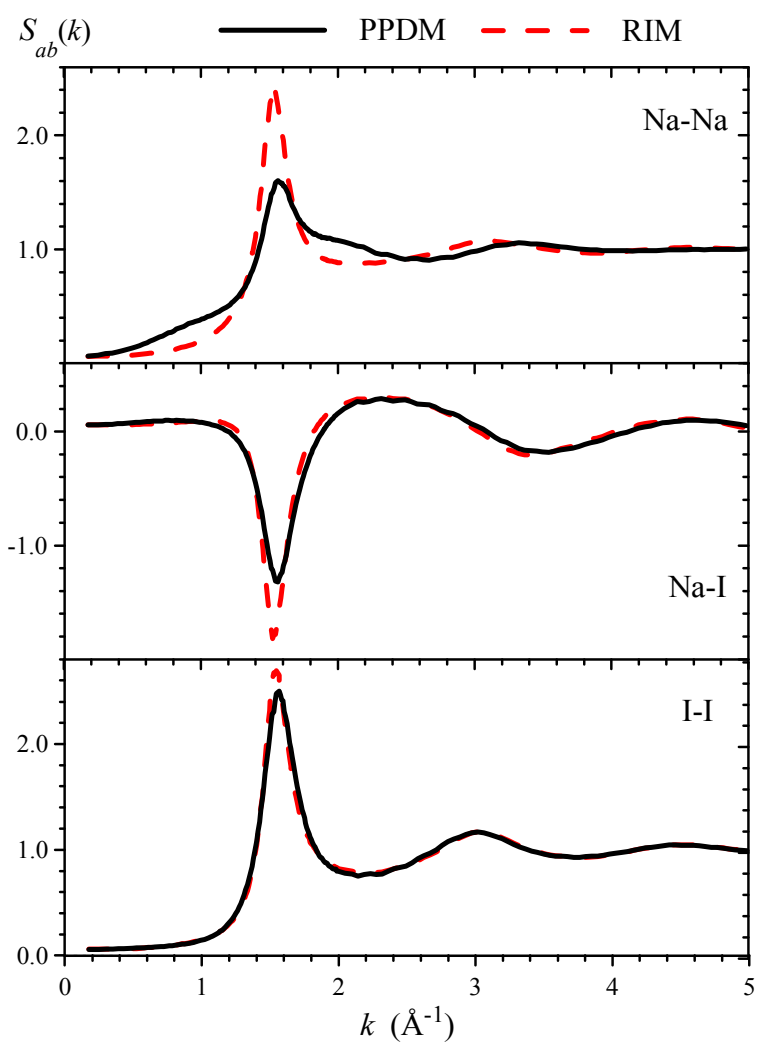

Fig. 3. Ashcroft-Langreth partial structure factors $S_{\mathrm{NaNa}}(k)$ (top), $S_{\mathrm{NaI}}(k)$ (middle), $S_{\mathrm{II}}(k)$ (bottom), for RIM (red dashed line) and PPDM (black solid line).

From the Ashcroft-Langreth partial structure factors $S_{a b}(k)$, which are plotted in Fig. 3 for RIM and PPDM, the structure factor of the intensity of neutron radiation coherently scattered is given by [11]

$$
S(k)=\left[b_{+}^{2} S_{++}(k)+b_{-}^{2} S_{--}(k)+2 b_{+} b_{-} S_{+-}(k)\right] /\left(b_{+}^{2}+b_{-}^{2}\right)
$$

where the coherent neutron scattering lengths are $b_{+}=b_{\mathrm{Na}}$ $=3.6 \mathrm{fm}$ and $b_{-}=b_{\mathrm{I}}=5.3 \mathrm{fm}$ [12]. The $S(k)$ for RIM and PPDM are compared in Fig. 4 with experimental data reported by McGreevy et al. [13] from inelastic neutron scattering. 


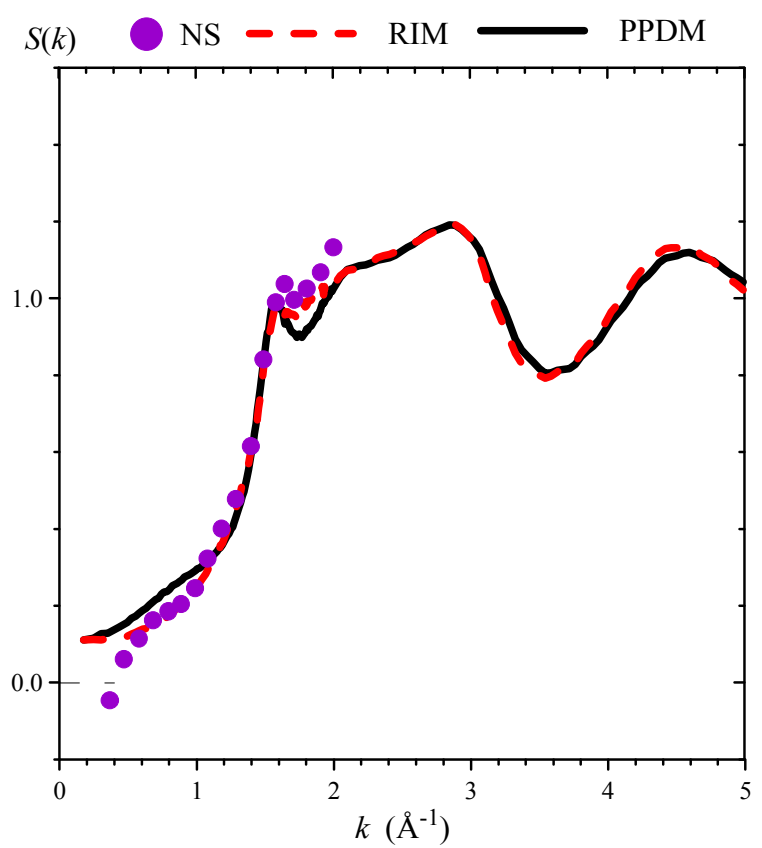

Fig. 4. Structure factor of the intensity of neutron radiation coherently scattered $S(k)$ for RIM (red dashed line), PPDM (black solid line), and neutron scattering data reported by McGreevy et al. [13]

\section{2 lonic transport properties}
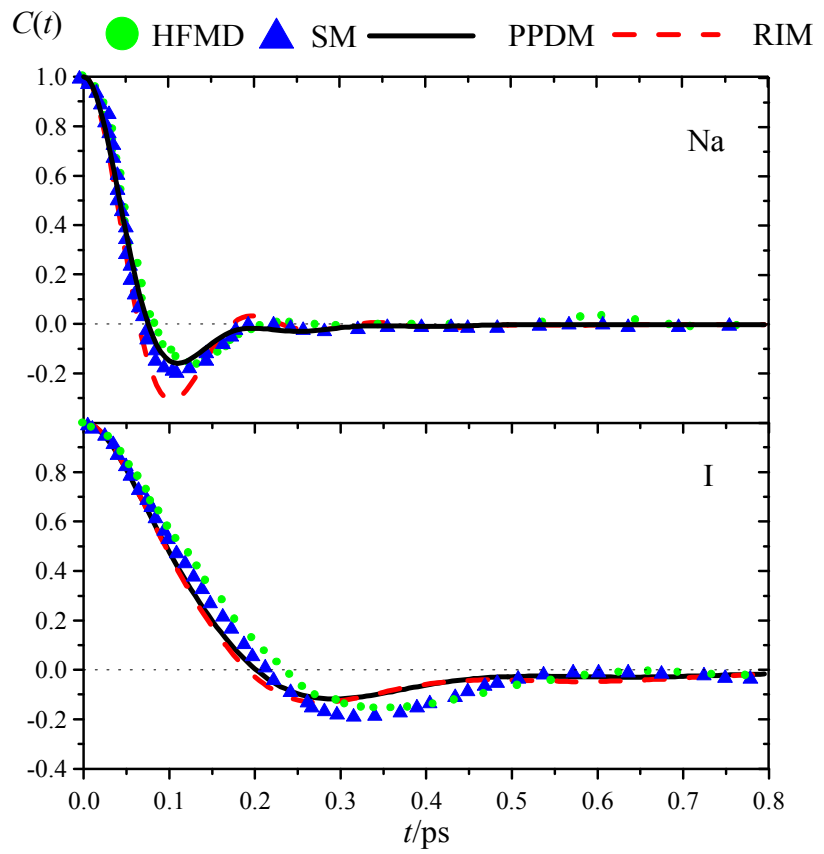

Fig. 5. Normalized velocity correlation functions $C_{a}(t)$ of $\mathrm{Na}$ (top) and I (bottom), for RIM (red dashed line), PPDM (black solid line), SM (blue dash and dot line) and HFMD (green circles).

Since cations are lighter than anions, the cation normalized self-velocity autocorrelation function, $C_{\mathrm{Na}}(t)$, exhibits a complete oscillation, while $C_{\mathrm{I}}(t)$ shows a slower decay and a weaker backscattering. The cations experience a rattling motion in the cage formed by neighboring heavier ions.
The $C_{\mathrm{Na}}(t)$ for PPDM presents a weaker backscattering than that of RIM. It indicates that the anionic cage effect is smaller when the anion polarization is allowed. The $C_{\mathrm{Na}}(t)$ for PPDM fits quite well that for HFMD and SM. The initial decay of $C_{\mathrm{I}}(t)$ for PPDM is faster than that for HFMD and SM.

The self-diffusion coefficients $D_{a}$ for PPDM and SM are in better agreement with experimental data than those for RIM and HFMD. The simulated results tend to underrate the experimental conductivity value.

Table 1. Ionic transport coefficients.

\begin{tabular}{|c|c|c|c|c|}
\hline & $T(\mathrm{~K})$ & $D_{\mathrm{Na}}($ fick $)$ & $D_{\mathrm{I}}($ fick $)$ & $\sigma(\Omega \cdot \mathrm{cm})^{-1}$ \\
\hline RIM & 995 & $\begin{array}{c}4.86 \\
\left(8.2^{a}-8.6^{b}\right)\end{array}$ & $\begin{array}{c}3.42 \\
\left(4.6^{a}-4.9^{b}\right)\end{array}$ & $\begin{array}{c}1.85 \\
\left(2.44^{\mathrm{c}}\right)\end{array}$ \\
\hline PPDM & 984 & $\begin{array}{c}8.67 \\
\left(8.0^{a}-8.4^{b}\right)\end{array}$ & $\begin{array}{c}4.05 \\
\left(4.5^{a}-4.7^{b}\right)\end{array}$ & $\begin{array}{c}1.75 \\
\left(2.41^{\mathrm{c}}\right)\end{array}$ \\
\hline SHM & 990 & $\begin{array}{c}8.1 \\
\left(8.1^{a}-8.5^{b}\right)\end{array}$ & $\begin{array}{c}4.0 \\
\left(4.6^{a}-4.8^{b}\right)\end{array}$ & \\
\hline HFMD & 995 & $\begin{array}{c}12.0 \\
\left(8.2^{a}-8.6^{b}\right)\end{array}$ & $\begin{array}{c}4.8 \\
\left(4.6^{a}-4.9^{b}\right)\end{array}$ & \\
\hline
\end{tabular}

$a, b$ and $c$ : experimental results in Refs. 14,15 and 16 .

\section{References}

1. M. Dixon and M. J. L. Sangster, J. Phys. C 9, 909 (1976)

2. M. J. L. Sangster and M. Dixon, Adv. Phys. 25, 247 (1976)

3. O. Alcaraz, V. Bitrián and J. Trullàs, J. Chem. Phys. 127, 154508 (2007)

4. J. Trullàs, O. Alcaraz, L. E. González, and M. Silbert, J. Phys. Chem. B 107, 282 (2003)

5. V. Bitrián and J. Trullàs, J. Phys. Chem. B 110, 7490 (2006)

6. V. Bitrián and J. Trullàs, J. Phys. Chem B 112, 1718 (2008)

7. N. Galamba, and B. J. Costa Cabral, J. Chem. Phys. 127, 094506 (2007)

8. J. E. Mayer, J. Chem. Phys. 1, 270 (1933)

9. M. Wilson and P. A. Madden, J. Phys.: Condens. Matter 5, 2867 (1993)

10. K. T. Tang and J. P. Toennies, J. Chem. Phys. 80, 3726 (1984)

11. M. P. Tosi, D. L. Price and M.L Saboungi Annu. Rev. Phys. Chem. 44, 173 (1993)

12. V. F. Sears, Neutron News 3, 26 (1992)

13. R. L. McGreevy, E. W. J. Mitchell and D. M. A. Margaca, J. Phys. C 17, 775 (1984)

14. J. O. M. Bockis, G. W. Hooper, Discuss. Faraday Soc. 32, 218 (1961)

15. S. B. Tricklebank, L. Nanis, and J. O. M. Bockris, J. Phys. Chem. 68, 58 (1964)

16. G. J. Janz et al. Molten Salts. Vol. 1 National Standard Reference Data Series (1968) 